Check for updates

Cite this: RSC Adv., 2017, 7, 24204

\title{
Effect of solvent polarity on the photophysical properties of chalcone derivatives $\dagger$
}

\author{
Rekha Kumari, (D) Anitha Varghese, (D) * Louis George and Sudhakar Y. N.
}

The absorption and fluorescence characteristics of (E)-3-(furan-2-yl)-1-(4-nitrophenyl)prop-2-en-1-one (FNPO), (E)-1-(4-aminophenyl)-3-(furan-2-yl)prop-2-en-1-one (AFPO) and (E)-3-(furan-2-yl)-1-(2hydroxyphenyl)prop-2-en-1-one (FHPO) were recorded in eighteen different solvents with increasing polarities at room temperature. The solvatochromic effects on absorption and fluorescence spectra have shown bathochromic shifts from non-polar to polar solvents for the reported molecules due to intramolecular charge transfer (ICT) interactions. It has indicated a large difference in dipole moment between electronically ground and excited states and the molecules were found to be more stabilized in singlet excited state than the ground state. The ground and excited state dipole moments of FNPO, AFPO and FHPO were determined experimentally by solvatochromic shift method using Bilot-Kawski, Lippert-Mataga, Bakhshiev, Kawski-Chamma-Viallet and Reichardt's microscopic solvent polarity functions. HOMO-LUMO energy values of FNPO, AFPO and FHPO were determined using cyclic voltammetry and compared with those values obtained by TD-DFT (B3LYP/6-311G(d,p)) method.

Received 10th February 2017 Accepted 22nd April 2017

DOI: $10.1039 / \mathrm{c} 7 \mathrm{ra01705g}$

rsc.li/rsc-advances
Various methods such as thermochromic shift method, ${ }^{25}$ Stark effect, ${ }^{26}$ fluorescence polarization, ${ }^{27}$ microwave conductivity, ${ }^{28}$ electric dichroism ${ }^{29}$ have been reported for the determination of dipole moments of fluorescent molecules. But, uses of these methods are limited as they are less sensitive and restricted to small molecules. Among these, solvatochromic shift method has been a widely accepted method for the determination of ground and singlet excited dipole moments because of linear correlation between spectral parameters and solvent polarity functions. ${ }^{30-32}$ There are considerable reports on the determination of ground and singlet excited state dipole moments using solvatochromic approaches for various chalcone derivatives such as substituted naphthalene triazole, thienyl, chloroquinoline, benzylidene benzosuberones, pyrrolnaphthalene, isatin based chalcone derivatives, indole based naphthalene and thiophene derivatives. ${ }^{33-39}$ However, no literature was found on the determination of ground and singlet excited state dipole moments for FNPO, AFPO and FHPO. The titled molecules were synthesized (Scheme 1) by Claisen Schmidt condensation reaction ${ }^{40}$ and characterized by IR, ${ }^{1} \mathrm{H}$ NMR, ${ }^{13} \mathrm{C}$ NMR and mass spectrometry. The molecular structures of the studied molecules have been depicted in Fig. 1.

The present study focuses on determination of ground and singlet excited state dipole moments of synthesized molecules using solvatochromic shift method. Bilot and Kawski, ${ }^{\mathbf{4 1 , 4 2}}$ Lippert-Mataga, ${ }^{43 a, b}$ Bakhshiev, ${ }^{\mathbf{4 4}}$ Kawski-Chamma-Viallet, ${ }^{\mathbf{4 5 a}, \boldsymbol{b}}$ and microscopic solvent polarity parameters $\left(E_{\mathrm{T}}^{\mathrm{N}}\right)^{\mathbf{4 6}}$ were used to determine the dipole moments experimentally. The specific and nonspecific interactions between solvents and solute molecules were studied using Kamlet-Abboud-Taft ${ }^{59}$ and Catalan solvent
Department of Chemistry, Christ University, Bangalore - 560 029, India. E-mail: anitha.varghese@christuniversity.in; Fax: +91 804012 9000; Tel: +91 8040129313 $\dagger$ Electronic supplementary information (ESI) available. See DOI: $10.1039 / \mathrm{c} 7 \mathrm{ra} 01705 \mathrm{~g}$ 
<smiles>O=Cc1ccco1</smiles>

furan-2-carbaldehyde<smiles>CC1CCCC1</smiles><smiles>[R1]c1ccc(C(C)=O)c([R1])c1</smiles>

Substituted Acetophenone

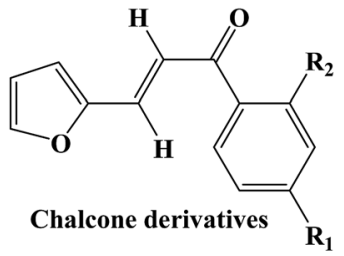

$$
\begin{aligned}
& \mathbf{R}_{1}=N_{2}, R_{2}=H \\
& R_{1}=N_{2}, R_{2}=H \\
& R_{1}=H, R_{2}=O H
\end{aligned}
$$

Scheme 1 Synthesis of different chalcone derivatives.

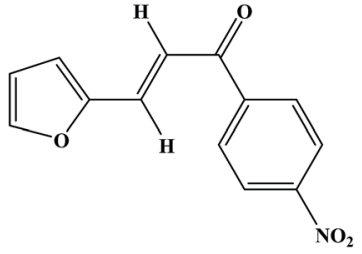

(E)-3-(furan-2-yl)-1-(4-nitrophenyl)prop-2-en-1-one (FNPO)

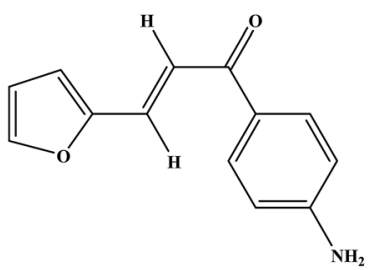

(E)-1-(4-aminophenyl)-3-(furan-2-yl)prop-2-en-1-one (AFPO)

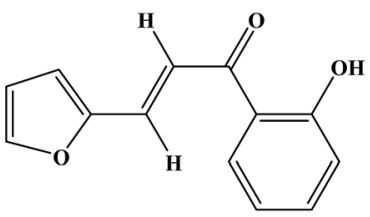

(E)-3-(furan-2-yl)-1-(2-hydroxyphenyl)prop-2-en-1-one (FHPO)

Fig. 1 Structure of chalcone derivatives.

polarity parameters. ${ }^{60}$ Gaussian 09 program $^{48}$ was used to complement the experimental results.

\section{Results and discussion}

\section{Spectral properties}

The electronic absorption and fluorescence spectra of FNPO, AFPO and FHPO were recorded in eighteen different solvents with increasing polarities from non-polar to polar at $10^{-5} \mathrm{M}$ concentration. The absorption and fluorescence spectra for FNPO have been presented in Fig. 2 and 3 respectively. Solvent parameters such as polarity, dielectric constant and polarizability play an important role to in inducing changes in intensity, position and size of absorption and fluorescence spectra. The absorption maxima of FNPO, AFPO and FHPO were found to be in the range of $352-380 \mathrm{~nm}, 348-378 \mathrm{~nm}$ and $350-379 \mathrm{~nm}$

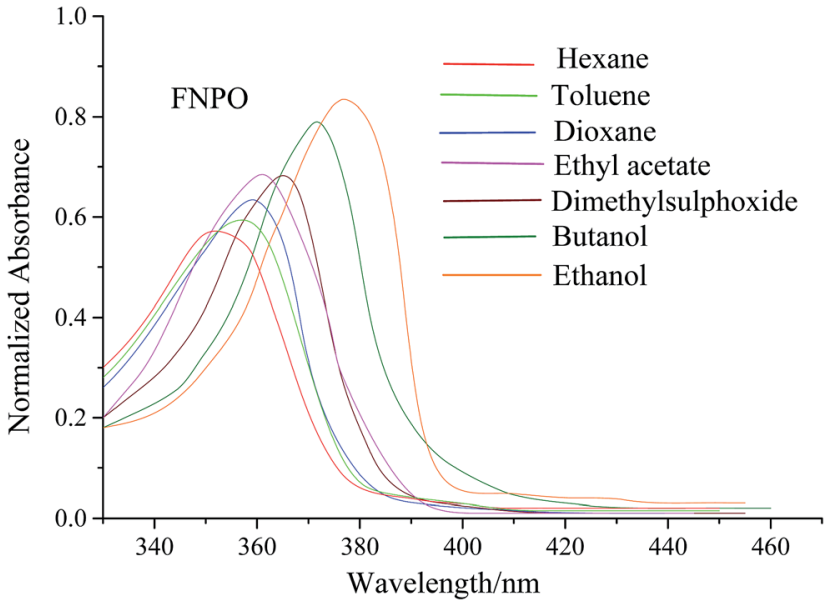

Fig. 2 Electronic absorption spectra of FNPO in different solvents.

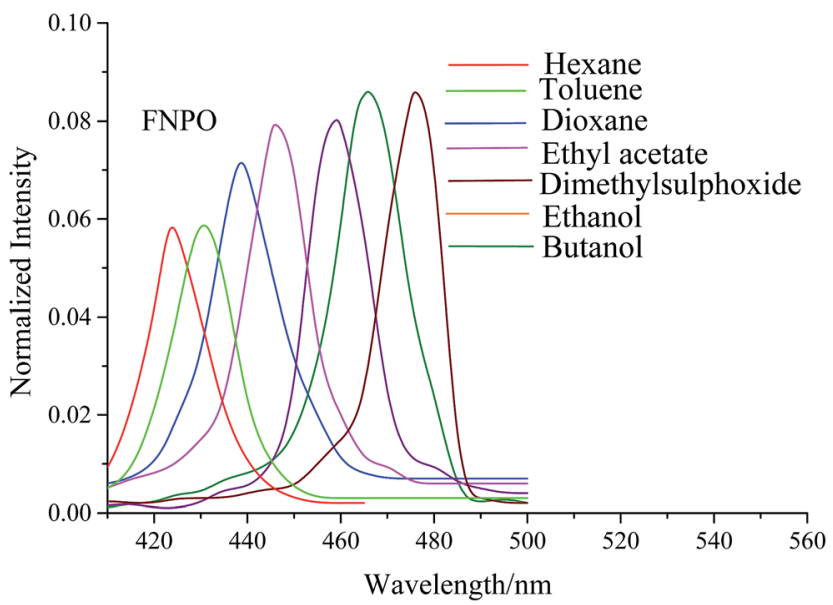

Fig. 3 Fluorescence spectra of FNPO in different solvents.

respectively whereas the fluorescence maxima were obtained in the range of 423-482 $\mathrm{nm}, 420-476 \mathrm{~nm}$ and 425-480 $\mathrm{nm}$. Upon increasing the solvent polarity, appreciable red shifts were observed in the fluorescence spectra of FNPO, AFPO and FHPO $\left(\Delta \lambda_{\mathrm{f}}=59 \mathrm{~nm}, 56 \mathrm{~nm}\right.$ and $55 \mathrm{~nm}$ respectively) when compared to their absorption spectra respectively $\left(\Delta \lambda_{\mathrm{a}}=38 \mathrm{~nm}, 30 \mathrm{~nm}\right.$ and $29 \mathrm{~nm}$ ). These results have confirmed that singlet excited state energy level was more stabilized than ground state energy 
Table 1 Spectroscopic data of FNPO in different solvents

\begin{tabular}{|c|c|c|c|c|c|c|c|}
\hline Solvents & $\lambda_{\mathrm{a}}(\mathrm{nm})$ & $\bar{\nu}_{\mathrm{a}}^{a}\left(\mathrm{~cm}^{-1}\right)$ & $\lambda_{\mathrm{f}}(\mathrm{nm})$ & $\bar{\nu}_{\mathrm{f}}^{b}\left(\mathrm{~cm}^{-1}\right)$ & $\Delta \bar{\nu}^{c}\left(\mathrm{~cm}^{-1}\right)$ & $\bar{\nu}_{\mathrm{a}}+\bar{\nu}_{\mathrm{f}} / 2^{d}\left(\mathrm{~cm}^{-1}\right)$ & $\bar{\nu}_{\mathrm{a}}+\bar{\nu}_{\mathrm{f}}\left(\mathrm{cm}^{-1}\right)$ \\
\hline Hexane & 352 & 28409 & 423 & 23640 & 4768 & 26024 & 52049 \\
\hline$p$-Xylene & 355 & 28169 & 428 & 23364 & 4804 & 25766 & 51533 \\
\hline Toluene & 356 & 28089 & 430 & 23255 & 4834 & 25672 & 51345 \\
\hline Ether & 357 & 28011 & 432 & 23148 & 4863 & 25579 & 51159 \\
\hline Diethylamine & 359 & 27855 & 437 & 22883 & 4971 & 25369 & 50738 \\
\hline 1,4-Dioxane & 360 & 27777 & 438 & 22831 & 4946 & 25304 & 50608 \\
\hline Chlorobenzene & 360 & 27777 & 440 & 22727 & 5050 & 25252 & 50505 \\
\hline Tetrahydrofuran & 362 & 27624 & 445 & 22471 & 5152 & 25048 & 50096 \\
\hline Ethyl acetate & 363 & 27548 & 446 & 22421 & 5126 & 24984 & 49969 \\
\hline Chloroform & 364 & 27472 & 447 & 22371 & 5101 & 24921 & 49843 \\
\hline Dichloromethane & 367 & 27247 & 453 & 22075 & 5172 & 24661 & 49323 \\
\hline Dimethyl formamide & 368 & 27173 & 458 & 21834 & 5339 & 24503 & 49007 \\
\hline Dimethylsulphoxide & 368 & 27173 & 459 & 21786 & 5387 & 24480 & 48960 \\
\hline Acetonitrile & 370 & 27027 & 462 & 21645 & 5382 & 24336 & 48672 \\
\hline Butanol & 372 & 26881 & 466 & 21459 & 5422 & 24170 & 48340 \\
\hline Propanol & 374 & 26737 & 470 & 21276 & 5461 & 24007 & 48014 \\
\hline Ethanol & 377 & 26525 & 476 & 21008 & 5516 & 23766 & 47533 \\
\hline Methanol & 380 & 26315 & 482 & 20746 & 5568 & 23531 & 47062 \\
\hline
\end{tabular}

${ }^{a}$ Absorption maxima. ${ }^{b}$ Fluorescence maxima. ${ }^{c}$ Stokes shift. ${ }^{d}$ Arithmetic mean of absorption maxima and fluorescence maxima.

level. The large fluorescence spectral shifts have suggested that the titled molecules were more polarized in the singlet excited state than in the ground state. ${ }^{45}$ Spectroscopic parameters of FNPO have been given in Table 1. Similar spectroscopic data for AFPO and FHPO in different solvents have also been calculated (Tables S1 and S2 ESI†). On increasing solvent polarities, Stokes shift values were found to vary from 4768 to 5568, 4926 to 5446 and 5042 to 5551 for FNPO, AFPO and FHPO respectively. These large differences in Stokes shift values $\left(800 \mathrm{~cm}^{-1}, 520 \mathrm{~cm}^{-1}\right.$ and $509 \mathrm{~cm}^{-1}$ for FNPO, AFPO and FHPO) indicated bathochromic shift and ICT due to $\pi \rightarrow \pi^{*}$ transition and confirmed that the molecules were more stabilized in polar solvents than in nonpolar ones. The bathochromic shifts have arisen due to the strong interaction of singlet excited state solute molecules with polar solvents leading to a large charge distribution between ground and singlet excited state of solute molecules.

The spectral bathochromic shifts and Stokes shifts were found to be more important in FNPO than in AFPO and FHPO, due to the greater electronic delocalization in the former compound than in the later ones, and to the respective, different electron-withdrawing and electron-donating effects of these substituents.

The quantum yield of FNPO, AFPO and FHPO were determined using single point method to study the solvent induced spectral changes and solute-solvent interactions. Anthracene in ethanol was used as a standard reference. The quantum yields have been determined using eqn (1) for FNPO, AFPO and FHPO in ethanolic medium and were found to be $0.12,0.18$ and 0.20 respectively. In case of FNPO, ICT state was more stabilized in polar environment due to effective hydrogen bonding between polar functional group of fluorophore and solvent molecules. This effectively quenched fluorescence emission and enhanced the non-radiative decay rate.

$$
\phi=\phi_{\text {std }} \times \frac{I_{\mathrm{S}}}{I_{\mathrm{R}}} \times \frac{\mathrm{OD}_{\mathrm{S}}}{\mathrm{OD}_{\mathrm{R}}} \times \frac{\eta_{\mathrm{S}}^{2}}{\eta_{\mathrm{R}}^{2}}
$$

where $\phi_{\text {std }}$ represented quantum yield of standard reference 0.27 (anthracene), ${ }^{49} I_{\mathrm{S}}$ and $I_{\mathrm{R}}$ were the integrated peak area of sample and standard reference, $\mathrm{OD}_{\mathrm{R}}$ and $\mathrm{OD}_{\mathrm{S}}$ were the optical densities of standard reference and sample, and $\eta_{\mathrm{S}}$ and $\eta_{\mathrm{R}}$ were refractive indices of solvent and standard reference respectively. The ratio of the squares of refractive indices was taken as 1 .

\section{Solvatochromic analysis for the estimation of ground and excited state dipole moments}

Solvatochromic behavior of FNPO, AFPO and FHPO were analyzed using linear correlation methods ${ }^{47}$ proposed by BilotKawski, Lippert-Mataga, Bakhshiev, Kawski-Chamma-Viallet and Reichardt. Spectroscopic properties have been correlated with different solvent polarity scales to estimate the experimental value of singlet excited state dipole moment. The solvent polarity functions $f(\varepsilon, n), \phi(\varepsilon, n), F_{1}(\varepsilon, n), F_{2}(\varepsilon, n)$ and $F_{3}(\varepsilon, n)$ were calculated using eqn (2), (3), (9), (10) and (11) respectively for eighteen different solvents of increasing polarities (given in Table 2). The ground state dipole moments $\left(\mu_{\mathrm{g}}\right)$ of FNPO, AFPO and FHPO were determined by quantum chemical calculation using Gaussian 09W program and were found to be $3.66 \mathrm{D}, 3.61$ $\mathrm{D}$ and $3.58 \mathrm{D}$ respectively. Theoretically obtained ground state dipole moment values were used for the calculation of singlet excited state dipole moment $\left(\mu_{\mathrm{e}}\right)$ by solvatochromic shift methods. Based on Bilot-Kawski correlation method, ground state dipole moments of the reported titled molecules were obtained as $5.87 \mathrm{D}, 5.72 \mathrm{D}$ and $7.13 \mathrm{D}$ respectively. Linear graphs of $\bar{\nu}_{\mathrm{a}}-\bar{\nu}_{\mathrm{f}} \nu s . f(\varepsilon, n), \bar{\nu}_{\mathrm{a}}+\bar{\nu}_{\mathrm{f}} \nu s . \phi(\varepsilon, n), \bar{\nu}_{\mathrm{a}}-\bar{\nu}_{\mathrm{f}} \nu s . F_{1}(\varepsilon, n), \bar{\nu}_{\mathrm{a}}-$ $\bar{\nu}_{\mathrm{f}} v s . F_{2}(\varepsilon, n), \bar{\nu}_{\mathrm{a}}+\bar{\nu}_{\mathrm{f}} / 2 v s . F_{3}(\varepsilon, n)$ and $\bar{\nu}_{\mathrm{a}}-\bar{\nu}_{\mathrm{f}} v s . E_{\mathrm{T}}^{\mathrm{N}}$ were plotted for FNPO, AFPO and FHPO. Solvatochromic plots for FNPO have been shown in Fig. 4. Slopes $m^{(2)}, m_{1}, m_{2}, m_{3}$ and $m_{4}$ obtained 
Table 2 Solvatochromic polarity functions $F_{1}(\varepsilon, n), F_{2}(\varepsilon, n), F_{3}(\varepsilon, n), \varepsilon, n, E_{T}^{N}, f(\varepsilon, n)$ and $\phi(\varepsilon, n)^{f}$

\begin{tabular}{|c|c|c|c|c|c|c|c|c|}
\hline Solvents & $F_{1}(\varepsilon, n)^{a}$ & $F_{2}(\varepsilon, n)^{b}$ & $F_{3}(\varepsilon, n)^{c}$ & $\varepsilon^{d}$ & $n^{e}$ & $E_{\mathrm{T}}^{\mathrm{N}}$ & $f(n, e)$ & $\phi(n, e)$ \\
\hline Hexane & -9.852 & -1.828 & 0.536 & 1.89 & 1.374 & 0 & 0.052 & 0.562 \\
\hline$p$-Xylene & 0.003 & 0.007 & 0.715 & 2.27 & 1.495 & 0.07 & 0.092 & 0.761 \\
\hline Ether & 0.164 & 0.370 & 0.689 & 4.27 & 1.353 & 0.11 & 0.417 & 0.898 \\
\hline Diethylamine & 0.126 & 0.283 & 0.694 & 3.6 & 1.386 & 0.14 & 0.338 & 0.863 \\
\hline 1,4-Dioxane & 0.021 & 0.043 & 0.624 & 2.22 & 1.422 & 0.16 & 0.108 & 0.681 \\
\hline Ethyl acetate & 0.200 & 0.492 & 0.778 & 6.08 & 1.372 & 0.22 & 0.544 & 1.050 \\
\hline Chloroform & 0.148 & 0.370 & 0.825 & 4.81 & 1.445 & 0.25 & 0.442 & 1.046 \\
\hline Dichloromethane & 0.218 & 0.594 & 0.905 & 9.08 & 1.424 & 0.31 & 0.660 & 1.235 \\
\hline Dimethyl formamide & 0.275 & 0.839 & 1.037 & 38.25 & 1.430 & 0.39 & 0.906 & 1.490 \\
\hline Dimethylsulphoxide & 0.263 & 0.842 & 1.105 & 47.24 & 1.477 & 0.44 & 0.921 & 1.566 \\
\hline Acetonitrile & 0.305 & 0.863 & 0.922 & 37.5 & 1.344 & 0.46 & 0.907 & 1.376 \\
\hline
\end{tabular}

${ }^{a}$ Lippert-Mataga solvent polarity function. ${ }^{b}$ Bakhshiev solvent polarity function. ${ }^{c}$ Kawski-Chamma-Viallet solvent polarity function. ${ }^{d} \varepsilon=$ dielectric constant. ${ }^{e} n=$ refractive index. ${ }^{f} E_{\mathrm{T}}^{\mathrm{N}}=$ microscopic solvent polarity function.

from $\bar{\nu}_{\mathrm{a}}+\bar{\nu}_{\mathrm{f}} v s . \phi(\varepsilon, n), \bar{\nu}_{\mathrm{a}}-\bar{\nu}_{\mathrm{f}} v s . F_{1}(\varepsilon, n), \bar{\nu}_{\mathrm{a}}-\bar{\nu}_{\mathrm{f}} v s . F_{2}(\varepsilon, n), \bar{\nu}_{\mathrm{a}}+\bar{\nu}_{\mathrm{f}} / 2$ vs. $F_{3}(\varepsilon, n)$ and $\bar{\nu}_{\mathrm{a}}-\bar{\nu}_{\mathrm{f}} v s . E_{\mathrm{T}}^{\mathrm{N}}$ were used to determine the singlet excited state $\left(\mu_{\mathrm{e}}\right)$ for FNPO, AFPO and FHPO.

From solvatochromic analysis of FNPO, AFPO and FHPO, correlation coefficient values were found to be greater than 0.90 , thereby indicating good linear square fitting of these correlations. ${ }^{50}$ The correlation graphs of $\bar{\nu}_{\mathrm{a}}-\bar{\nu}_{\mathrm{f}} v s . f(\varepsilon, n)$ and $\bar{\nu}_{\mathrm{a}}+\bar{\nu}_{\mathrm{f}} v s$. $\phi(\varepsilon, n)$ were plotted according to Bilot-Kawski method. The slopes $m^{(1)}$ and $m^{(2)}$ obtained from Bilot-Kawski plots based on eqn (2) and (3) have been used in eqn (4) and (5) to calculate ground state dipole moment $\left(\mu_{\mathrm{g}}\right)$ and singlet excited state dipole moment $\left(\mu_{\mathrm{e}}\right)$ respectively. The ground $\left(\mu_{\mathrm{g}}\right)$ and singlet excited state dipole moments $\left(\mu_{\mathrm{e}}\right)$ were found to be $5.87 \mathrm{D}$ and $8.05 \mathrm{D}$ (FNPO), 5.72D and 7.28D (AFPO) and 7.13D and 8.42D (FHPO) respectively and have been tabulated in Table 4 . As can be seen in this table, significant differences were found for the singlet excited state dipole moment $\left(\mu_{\mathrm{e}}\right)$ values of chalcone derivatives, depending on the type of solvatochromic relationship. These differences in the $\mu_{\mathrm{e}}$ values are very probably due to a number of assumptions and approximations utilized in these solvatochromic methods, as pointed out in the literature. ${ }^{51-56}$ The difference in dipole moment between ground and singlet excited states suggests that the molecules have considerable charge distribution in singlet excited state and hence play an important role in ICT process.

$$
\begin{gathered}
\bar{\nu}_{\mathrm{a}}-\bar{\nu}_{\mathrm{f}}=m^{(1)} f(\varepsilon, n)+\mathrm{constant} \\
\bar{\nu}_{\mathrm{a}}+\bar{\nu}_{\mathrm{f}}=-m^{(2)} \phi(\varepsilon, n)+\mathrm{constant}
\end{gathered}
$$

where, $f(\varepsilon, n)=\left[\frac{\varepsilon-1}{\varepsilon+2}+\frac{n^{2}-1}{n^{2}+2}\right] \frac{\left(2 n^{2}+1\right)}{\left(n^{2}+2\right)}$ and $\phi(\varepsilon, n)=[f(\varepsilon, n)$ $+2 g(n)] ; g(n)=\frac{3}{2}\left[\frac{\left(n^{4}-1\right)}{\left(n^{2}+2\right)^{2}}\right]$.
The dielectric constant $(\varepsilon)$, refractive index $(n)$ and microscopic solvent polarities have been taken from literature. ${ }^{57}$

$$
\begin{gathered}
\mu_{\mathrm{g}}=\frac{m^{(2)}-m^{(1)}}{2}\left(\frac{h c a^{3}}{2 m^{(1)}}\right)^{\frac{1}{2}} \\
\mu_{\mathrm{e}}=\frac{m^{(2)}+m^{(1)}}{2}\left(\frac{h c a^{3}}{2 m^{(1)}}\right)^{\frac{1}{2}} \\
\bar{\nu}_{\mathrm{a}}-\bar{\nu}_{\mathrm{f}}=m_{1} F_{1}(\varepsilon, n)+\text { constant } \\
\bar{\nu}_{\mathrm{a}}-\bar{\nu}_{\mathrm{f}}=-m_{2} F_{2}(\varepsilon, n)+\text { constant } \\
\frac{\bar{\nu}_{\mathrm{a}}+\bar{\nu}_{\mathrm{f}}}{2}=m_{3} F_{3}(\varepsilon, n)+\text { constant } \\
F_{1}(\varepsilon, n)=\frac{\varepsilon-1}{2 \varepsilon+1}-\frac{n^{2}-1}{2 n^{2}+1} \\
F_{2}(\varepsilon, n)=\left[\frac{\varepsilon-1}{\varepsilon+2}+\frac{n^{2}-1}{n^{2}+2}\right] \frac{\left(2 n^{2}+1\right)}{\left(n^{2}+2\right)} \\
{\left[\frac{\left(2 n^{2}+1\right)}{2\left(n^{2}+2\right)}\left(\frac{\varepsilon-1}{\varepsilon+2}+\frac{n^{2}-1}{n^{2}+2}\right)+\frac{3\left(n^{4}-1\right)}{2\left(n^{2}+2\right)^{2}}\right]}
\end{gathered}
$$

The slopes $m_{1}$ (Lippert-Mataga), $m_{2}$ (Bakhshiev), $m_{3}$ (KawskiChamma-Viallet) and $m_{4}$ (Reichardt) were obtained from the linear plots of $\left(\bar{\nu}_{\mathrm{a}}-\bar{\nu}_{\mathrm{f}}\right)$ vs. $F_{1}(\varepsilon, n)$ (Lippert-Mataga), $F_{2}(\varepsilon, n)$ (Bakhshiev), $\left(\bar{\nu}_{\mathrm{a}}+\bar{\nu}_{\mathrm{f}}\right) / 2$ vs. $F_{3}(\varepsilon, n)$ (Kawski-Chamma-Viallet) and $\left(\bar{\nu}_{\mathrm{a}}-\bar{\nu}_{\mathrm{f}}\right) v s . E_{\mathrm{T}}^{\mathrm{N}}(\varepsilon, n)$ (Reichardt), plotted for FNPO, AFPO and FHPO based on eqn (6), (7), (8), and (19). The solvatochromic graphs for FNPO have been presented in Fig. 4. Similar solvatochromic linear plots for AFPO and FHPO have also been plotted (Fig. S3 and $\mathrm{S} 4 \mathrm{ESI} \dagger)$. The obtained slopes, intercept and correlation 

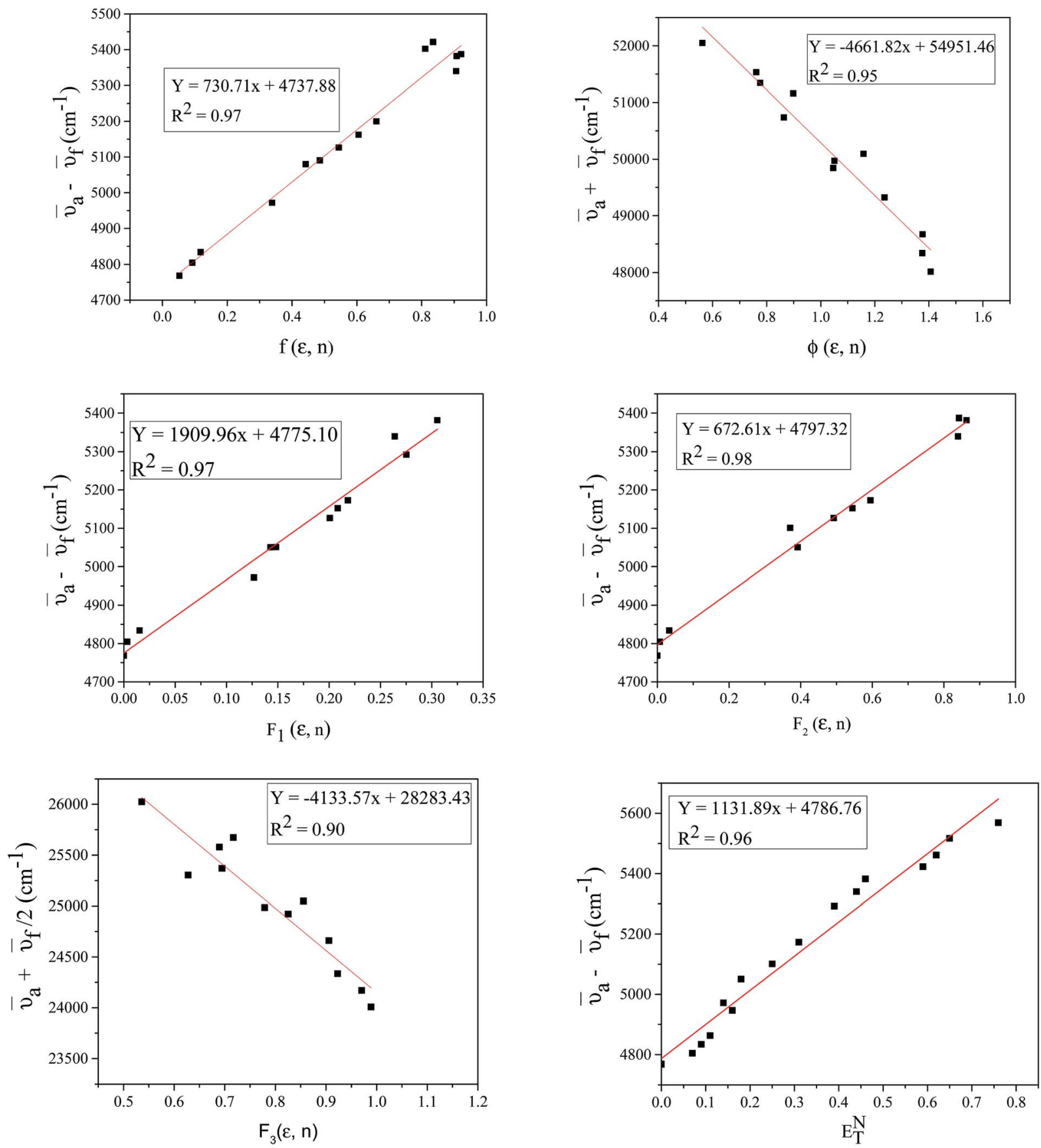

Fig. 4 Solvatochromic linear plots of $\bar{\nu}_{a}-\bar{\nu}_{f} v s$. $f(\varepsilon, n), \bar{\nu}_{a}+\bar{\nu}_{f} v s . \phi(\varepsilon, n)$ (Bilot-Kawski), $\left(\bar{\nu}_{a}-\bar{\nu}_{f}\right) v s . F_{1}(\varepsilon, n)\left(\right.$ Lippert-Mataga), $F_{2}(\varepsilon, n)(B a k h s h i e v),\left(\bar{\nu}_{a}+\right.$ $\left.\bar{\nu}_{f}\right) / 2$ vs. $F_{3}(\varepsilon, n)$ (Kawski-Chamma-Viallet) and $\left(\bar{\nu}_{a}-\bar{\nu}_{f}\right) v s . E_{\mathrm{T}}^{N}(\varepsilon, n)$ (Reichardt) for FNPO.

coefficient for FNPO, AFPO and FHPO have been represented in Table 3. Using these slope values, corresponding singlet excited state dipole moments $\left(\mu_{\mathrm{e}}\right)$ were calculated for FNPO, AFPO and FHPO using eqn (12)-(14) and (20) and were tabulated in Table $4{ }^{51-56}$ The singlet excited state dipole moment values were found to be higher than that of ground state thereby confirming that the molecules were more polarized in singlet excited state than in the ground state. It also suggests that the molecules have strong solute-solvent interaction causing large charge distribution in the singlet excited state. The ratio of singlet excited state dipole moment and ground state dipole moment $\left(\mu_{\mathrm{e}} / \mu_{\mathrm{g}}\right)$ and angle $(\phi)$ between them were estimated using eqn (17) and (18). The Onsager radii $(a)$ for FNPO, AFPO and FHPO were calculated using Edward's increment method ${ }^{58}$ (eqn (21)) and were found to be $3.66,3.61$ and 3.58 respectively.

$$
\begin{aligned}
& m_{1}=\frac{2\left(\mu_{\mathrm{e}}-\mu_{\mathrm{g}}\right)^{2}}{h c a^{3}} \\
& m_{2}=\frac{2\left(\mu_{\mathrm{e}}-\mu_{\mathrm{g}}\right)^{2}}{h c a^{3}}
\end{aligned}
$$


Table 3 Statistical treatment of correlation of solvents spectral shifts of FNPO, AFPO and FHPO molecules

\begin{tabular}{|c|c|c|c|c|c|}
\hline Compounds & Method & Slope $(m)$ & Intercept & Correlation coefficient $\left(r^{2}\right)$ & $N$ \\
\hline \multirow[t]{5}{*}{ FNPO } & Bilot-Kawski & $m^{(1)}=730.71$ & 4737.88 & 0.97 & 14 \\
\hline & Bilot-Kawski & $m^{(2)}=-4661.82$ & 54951.46 & 0.95 & 12 \\
\hline & Bakhshiev & $m_{2}=672.61$ & 4797.32 & 0.98 & 11 \\
\hline & Kawski-Chamma-Viallet & $m_{3}=-4133.57$ & 28283.43 & 0.90 & 12 \\
\hline & Reichardt & $m_{4}=1131.89$ & 4786.76 & 0.96 & 16 \\
\hline & Lippert-Mataga & $m_{1}=1604.28$ & 4901.23 & 0.95 & 16 \\
\hline & Bakhshiev & $m_{2}=564.10$ & 4919.38 & 0.95 & 18 \\
\hline & Kawski-Chamma-Viallet & $m_{3}=-5542.07$ & 29639.14 & 0.90 & 10 \\
\hline & Reichardt & $m_{4}=546.16$ & 5030.67 & 0.93 & 12 \\
\hline \multirow[t]{2}{*}{ FHPO } & Bilot-Kawski & $m^{(1)}=405.64$ & 5033.37 & 0.93 & 13 \\
\hline & Bilot-Kawski & $m^{(2)}=-4884.37$ & 55240.41 & 0.92 & 13 \\
\hline
\end{tabular}

Table 4 Ground and excited state dipole moments of FNPO, AFPO and FHPO ${ }^{a}$

\begin{tabular}{|c|c|c|c|c|c|c|c|c|c|c|c|c|}
\hline Compound & Radius $(\AA)$ & $\mu_{\mathrm{g}}{ }^{b}(\mathrm{D})$ & $\mu_{\mathrm{g}}{ }^{c}(\mathrm{D})$ & $\mu_{\mathrm{e}}^{d}(\mathrm{D})$ & $\mu_{\mathrm{e}}^{e}(\mathrm{D})$ & $\mu_{\mathrm{e}}^{f}(\mathrm{D})$ & $\mu_{\mathrm{e}}^{g}(\mathrm{D})$ & $\mu_{\mathrm{e}}^{h}(\mathrm{D})$ & $\Delta \mu^{i}(\mathrm{D})$ & $\Delta \mu^{j}(\mathrm{D})$ & $\left(\mu_{\mathrm{e}} / \mu_{\mathrm{g}}\right)^{k}$ & $\phi^{l}$ \\
\hline FNPO & 3.66 & 6.90 & 5.87 & 8.05 & 10.43 & 8.92 & 12.09 & 8.20 & 2.18 & 1.29 & 1.38 & 0 \\
\hline AFPO & 3.61 & 5.04 & 5.72 & 7.28 & 7.64 & 6.58 & 9.87 & 5.92 & 2.01 & 0.87 & 1.22 & 0 \\
\hline
\end{tabular}

${ }^{a}$ Debye (D) $=3.33564 \times 10^{-30} \mathrm{~cm}=10^{-18}$ esu cm. ${ }^{b}$ The theoretically calculated ground-state dipole moment by Gaussian software. ${ }^{c}$ The ground state dipole moment calculated from Bilot-Kawski equation. ${ }^{d}$ The excited state dipole moment calculated from Bilot-Kawski equation. ${ }^{e}$ The excited state dipole moment calculated from Lippert-Mataga equation. ${ }^{f}$ The excited state dipole moment calculated from Bakhshiev's equation. ${ }^{g}$ The excited state dipole moment calculated from Kawski-Chamma-Viallet's equation. ${ }^{h}$ The excited-state dipole moment calculated from microscopic solvent polarity parameter equation. ${ }^{i}$ The change in dipole moments for $\mu_{\mathrm{e}}$ and $\mu_{\mathrm{g}}$ obtained from Bilot-Kawski method. ${ }^{j}$ The change in dipole moments calculated from eqn (20). ${ }^{k}$ The ratio of excited state dipole moment and ground state dipole moment calculated from eqn (17). ${ }^{l}$ The angle between ground state dipole moment and excited state dipole moment.

$$
\begin{gathered}
m_{3}=\frac{2\left(\mu_{\mathrm{e}}^{2}-\mu_{\mathrm{g}}^{2}\right)}{h c a^{3}} \\
\mu_{\mathrm{g}}=\frac{m_{3}-m_{2}}{2}\left(\frac{h c a^{3}}{2 m_{2}}\right)^{\frac{1}{2}} \\
\mu_{\mathrm{e}}=\frac{m_{3}+m_{2}}{2}\left(\frac{h c a^{3}}{2 m_{2}}\right)^{\frac{1}{2}} \\
\mu_{\mathrm{e}}=\frac{m_{2}+m_{3}}{m_{3}-m_{2}} \mu_{\mathrm{g}} \quad \text { for } m_{3}>m_{2} \\
\cos \phi=\frac{1}{2 \mu_{\mathrm{g}} \mu_{\mathrm{e}}}\left[\left(\mu_{\mathrm{g}}{ }^{2}+\mu_{\mathrm{e}}^{2}\right)-\frac{m_{3}}{m_{2}}\left(\mu_{\mathrm{e}}{ }^{2}-\mu_{\mathrm{g}}{ }^{2}\right)\right] \\
\overline{\nu_{\mathrm{a}}}-\overline{\nu_{\mathrm{f}}}=m_{4} E_{\mathrm{T}}^{\mathrm{N}}+\text { const } \\
m_{4}=11307.6\left[\left(\frac{\Delta \mu}{\Delta \mu_{\mathrm{B}}}\right)^{2}\left(\frac{a_{\mathrm{B}}}{a}\right)^{3}\right] \\
\Delta \mu=\left(\mu_{\mathrm{e}}-\mu_{\mathrm{g}}\right)=\sqrt{\frac{m_{4} 81}{11307.6(6.2 / a)^{3}}}
\end{gathered}
$$$$
\text { where, } m_{4}=11307.6\left[\left(\frac{\Delta \mu}{\Delta \mu_{\mathrm{B}}}\right)^{2}\left(\frac{a_{\mathrm{B}}}{a}\right)^{3}\right]
$$

$$
a=\left[\frac{3 M}{4 \pi \delta N_{\mathrm{A}}}\right]^{1 / 3}
$$

where $M, \delta$ and $N_{\mathrm{A}}$ are molecular weight, density and Avogadro's no. of solute molecules.

\section{Multi-parametric correlations}

The multiple linear regression analyses proposed by KamletAbboud-Taft ${ }^{59}$ and Catalan ${ }^{60}$ have been used to correlate absorption maxima, emission maxima and Stokes shift energies. ${ }^{61-63}$

$$
\begin{aligned}
& y=y_{0}+a_{\pi^{*}} \pi^{*}+b_{\alpha} \alpha+c_{\beta} \beta \\
& y=y_{0}+a_{\mathrm{SP}} \mathrm{SP}+b_{\mathrm{SdP}} \mathrm{SdP}+c_{\mathrm{SA}} \mathrm{SA}+d_{\mathrm{SB}} \mathrm{SB}
\end{aligned}
$$

where $y$ and $y_{0}$ were solvent dependent physiochemical property and gaseous statistical quantity respectively. Independent solvent parameters such as $\pi^{*}, \alpha, \beta, \mathrm{SP}, \mathrm{SdP}, \mathrm{SA}$ and SB quantify solute-solvent interactions whereas $a_{\pi^{*}}, b_{\alpha}, c_{\beta}, a_{\mathrm{SP}}, b_{\mathrm{SdP}}, c_{\mathrm{SA}}$ and $d_{\mathrm{SB}}$ reflect the sensitivity of physical property $y$ in a given solvent with various solvent parameters. The Kamlet-Abboud-Taft and 
Catalan solvent parameters for AFPO and FHPO (Tables S5 and S6 ESI $\dagger$ ) were used to measure solvent's ability to stabilize dipole or charge of molecules through nonspecific or specific interactions. Nonspecific interactions were measured using solvent's dielectric parameters such as $\pi^{*}$, SP (solvent polarity) and SdP (solvent dipolarity) whereas specific interactions were measured using $\alpha$ or SA (hydrogen bond donor strength) and $\beta$ or SB (hydrogen bond donor strength) according to eqn (22) and (23).

The results obtained from multiple linear correlation of Kamlet-Abboud-Taft and Catalan equations have been shown in Tables 5 and 6 . The results of Kamlet-Abboud-Taft and Catalan solvent scales have confirmed bathochromic shift in absorption and fluorescence spectra on increasing polarities due to negative values for all the parameters in eqn (22) and (23). Large values of dipolarity/polarizability $\left(\pi^{*}\right)$ and solvent acidity $(\alpha)$ correlation regression coefficients reflect their higher contribution towards bathochromic shifts and solute-solvent interactions. The changes in absorption and fluorescence spectra were controlled mainly by dipolarity/polarizability and hydrogen bond donating ability of the solvents. Fluorescence spectra have indicated better correlation between KamletAbboud-Taft solvent scales and spectroscopic parameters than the absorption spectra. It can be confirmed that the electronic structure of ground and singlet excited state of FNPO, AFPO and FHPO differ significantly and the singlet excited state was more polarized than the ground state. Hence the dipole moments of singlet excited state of FNPO, AFPO and FHPO were found to be more than the ground state dipole moments.

In the case of Catalan solvent scales, the correlation regression coefficient values of polarizability/dipolarity and acidity were found to be more than the basicity of solvents. This confirms that polarizability and acidity of solvents play an important role in the bathochromic shifts of absorption and fluorescence spectra and solute-solvent interactions. Moreover, the large correlation coefficient values for fluorescence spectra have confirmed that the electronic structure of ground and singlet excited states of FNPO, AFPO and FHPO differ significantly. Hence, the dipole moment values of singlet excited state of FNPO, AFPO and FHPO were found to be more than the ground state dipole moments. This indicates that the titled molecules have both nonspecific and specific interactions.

\section{Theoretical investigations}

Quantum chemical calculations. The molecular geometry of FNPO, AFPO and FHPO were optimized and their HOMOLUMO energy values were determined by TD-DFT (B3LYP/6$311 \mathrm{G}(\mathrm{d}, \mathrm{p}))$ and have been depicted in Fig. 5 and 6 respectively. The frontier molecular orbitals (HOMO and LUMO orbital) play a vital role in understanding the electronic structures and transitions due to their electron donating and accepting abilities. The energy gap values were calculated between HOMO and LUMO energy levels for the reported molecules and have been presented in Fig. 6, which helps to describe the chemical reactivity, chemical softness and hardness, thermal and kinetic stability, optical polarizability as well ICT transition in the molecules. ${ }^{64}$

HOMO, LUMO and the energy gap values for FNPO, AFPO and FHPO have been tabulated in Table 7. The low values of energy gap suggest that the molecules were more reactive, soft

Table 5 Estimated coefficients $\left(y_{0}, a_{\pi^{*}}, b_{\alpha,} c_{\beta}\right)$, their errors and correlation coefficients $\left(r^{2}\right)$ for multi-linear correlation analysis of $\bar{\nu}_{\mathrm{a}}, \bar{\nu}_{\mathrm{f}}, \Delta \bar{\nu}$ of FNPO, AFPO and FHPO as a function of Kamlet-Abboud-Taft solvent scale (eqn (22))

\begin{tabular}{|c|c|c|c|c|c|c|c|}
\hline Compound & Parameters & $y_{0}$ & $a_{\pi^{*}}$ & $b_{\alpha}$ & $c_{\beta}$ & $r^{2}$ & $N$ \\
\hline \multirow[t]{2}{*}{ FNPO } & $\bar{\nu}_{\mathrm{a}}\left[\mathrm{cm}^{-1}\right]$ & $28410 \pm 95$ & $-(944 \pm 140)$ & $-(1159 \pm 125)$ & $-(478 \pm 137)$ & 0.9305 & 18 \\
\hline & $\bar{\nu}_{\mathrm{f}}\left[\mathrm{cm}^{-1}\right]$ & $23719 \pm 42$ & $-(1400 \pm 211)$ & $-(1564 \pm 188)$ & $-(785 \pm 205)$ & 0.9241 & 18 \\
\hline \multirow[t]{3}{*}{ AFPO } & $\bar{\nu}_{\mathrm{a}}\left[\mathrm{cm}^{-1}\right]$ & $28751 \pm 128$ & $-(1019 \pm 190)$ & $-(1318 \pm 169)$ & $-(647 \pm 185)$ & 0.9075 & 18 \\
\hline & $\bar{\nu}_{\mathrm{f}}\left[\mathrm{cm}^{-1}\right]$ & $23870 \pm 151$ & $-(1375 \pm 225)$ & $-(1498 \pm 200)$ & $-(868 \pm 219)$ & 0.9137 & 18 \\
\hline & $\Delta \bar{\nu}\left[\mathrm{cm}^{-1}\right]$ & $4856 \pm 48$ & $357 \pm 71$ & $183 \pm 63$ & $253 \pm 69$ & 0.8060 & 18 \\
\hline & $\Delta \bar{\nu}\left[\mathrm{cm}^{-1}\right]$ & $5007 \pm 33$ & $237 \pm 49$ & $299 \pm 44$ & $156 \pm 48$ & 0.8856 & 18 \\
\hline
\end{tabular}

Table 6 Estimated coefficients $\left(y_{0}, a_{\mathrm{SP}}, b_{\mathrm{SdP}}, c_{\mathrm{SB}}, d_{\mathrm{SA}}\right)$, their errors and correlation coefficients $\left(r^{2}\right)$ for multi-linear correlation analysis of $\bar{\nu}_{\mathrm{a}}, \bar{\nu}_{\mathrm{f}}, \Delta \bar{\nu}$ of FNPO, AFPO and FHPO as a function of Catalan four-parameter solvent scale (eqn (23))

\begin{tabular}{|c|c|c|c|c|c|c|c|c|}
\hline Compound & Parameters & $y_{0}$ & $a_{\mathrm{SP}}$ & $b_{\mathrm{SdP}}$ & $c_{\mathrm{SB}}$ & $d_{\mathrm{SA}}$ & $r^{2}$ & $N$ \\
\hline \multirow[t]{2}{*}{ FNPO } & $\bar{\nu}_{\mathrm{a}}\left[\mathrm{cm}^{-1}\right]$ & $30135 \pm 363$ & $-(2156 \pm 460)$ & $-(1315 \pm 225)$ & $-(304 \pm 203)$ & $-(2045 \pm 10)$ & 0.8968 & 18 \\
\hline & $\bar{\nu}_{\mathrm{f}}\left[\mathrm{cm}^{-1}\right]$ & $26009 \pm 489$ & $-(2819 \pm 618)$ & $-(1968 \pm 302)$ & $-(466 \pm 273)$ & $-(2787 \pm 17)$ & 0.9096 & 18 \\
\hline \multirow[t]{3}{*}{ AFPO } & $\bar{\nu}_{\mathrm{a}}\left[\mathrm{cm}^{-1}\right]$ & $30928 \pm 476$ & $-(2656 \pm 602)$ & $-(1517 \pm 294)$ & $-(535 \pm 265)$ & $-(2263 \pm 06)$ & 0.8720 & 18 \\
\hline & $\bar{\nu}_{\mathrm{f}}\left[\mathrm{cm}^{-1}\right]$ & $26149 \pm 559$ & $-(2793 \pm 708)$ & $-(1921 \pm 346)$ & $-(599 \pm 312)$ & $-(2656 \pm 77)$ & 0.8813 & 18 \\
\hline & $\Delta \bar{\nu}\left[\mathrm{cm}^{-1}\right]$ & $4751 \pm 127$ & $92 \pm 161$ & $473 \pm 79$ & $88 \pm 72$ & $352 \pm 109$ & 0.8622 & 18 \\
\hline & $\Delta \bar{\nu}\left[\mathrm{cm}^{-1}\right]$ & $4601 \pm 88$ & $470 \pm 111$ & $385 \pm 54$ & $83 \pm 49$ & $514 \pm 75$ & 0.9197 & 18 \\
\hline
\end{tabular}



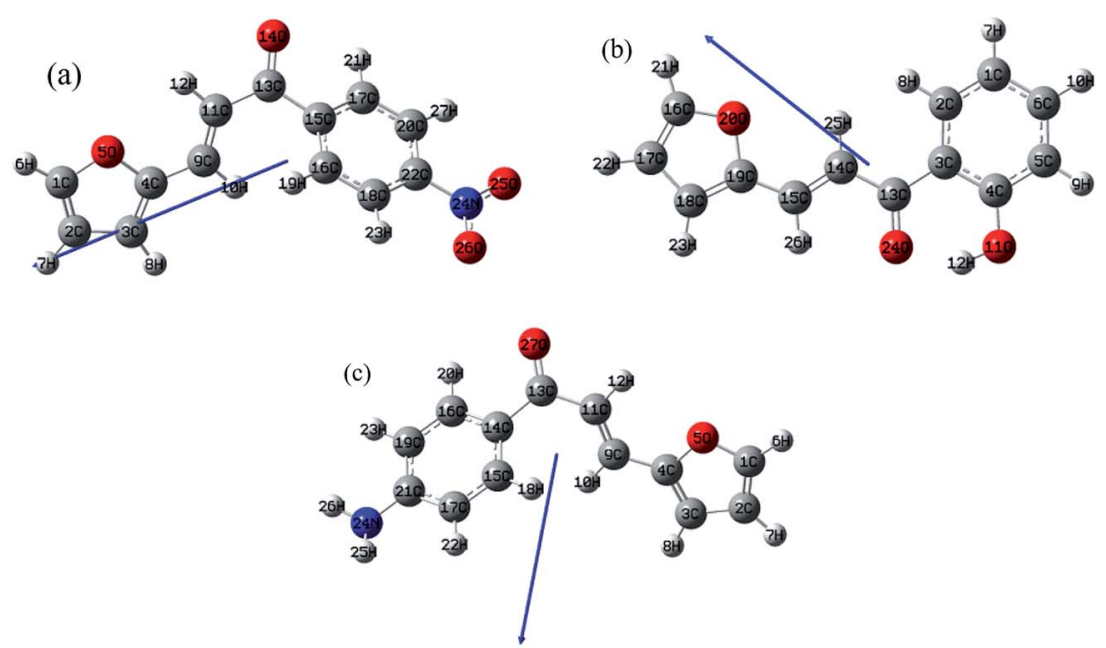

Fig. 5 The optimized geometry, numbering system, vector of the dipole moments of FNPO (a), FHPO (b) and AFPO (c) using (B3LYP/6$311 G(d, p))$.

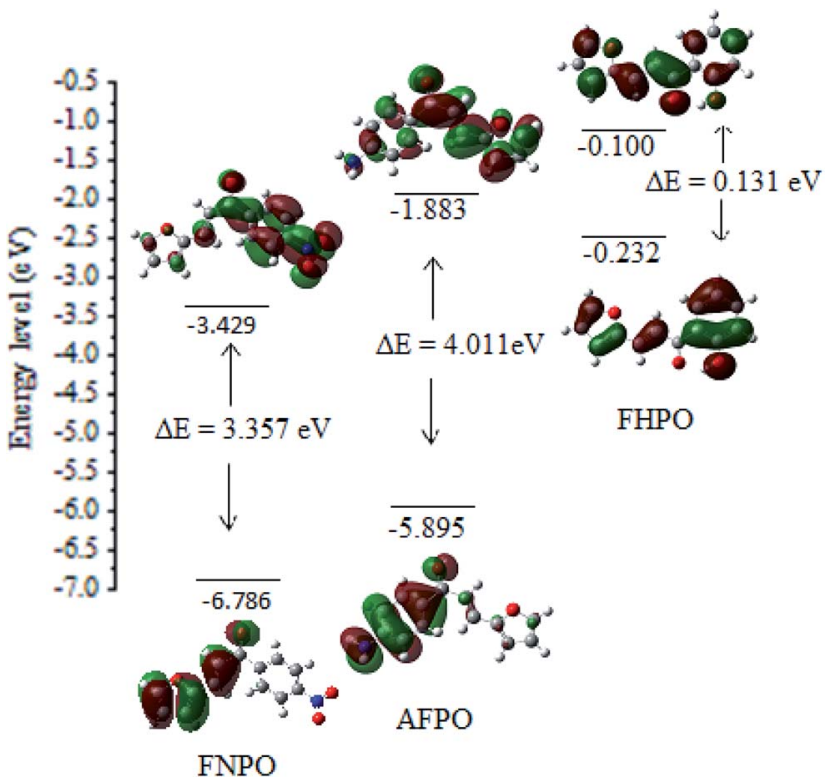

Fig. 6 Molecular orbital diagram of FNPO, AFPO and FHPO with HOMO-LUMO density plot and $\triangle E_{\text {HOMO-LUMO }}$ calculated at (B3LYP/6-311G(d,p)) level of theory.

and have easier $\pi \rightarrow \pi^{*}$ electronic transition. The positive and negative phases of HOMO-LUMO orbitals have been represented in red and green regions. In FNPO, the HOMO orbitals were localized in fural part whereas LUMO orbitals are localized from carbonyl group to nitro group. Therefore, delocalizaion of electrons occur from fural to carbonyl and nitro group of phenyl ring. This can be confirmed by Mulliken charge density plot of FNPO. The electron densities of O5, O14, $\mathrm{O} 25$ and $\mathrm{O} 26$ atoms were found to be $-0.4724,-0.3534,-0.1447$ and -0.2887 respectively, thereby indicating the oxygen atom of fural has more electron density than that of nitro group. HOMO orbitals of AFPO and FHPO were localized in amine and hydroxyl group of phenyl ring whereas LUMO orbitals were localized in
Table 7 Ground state molecular orbital energies ( $E_{\text {HOMO }}, E_{\mathrm{LUMO}}$ ) and

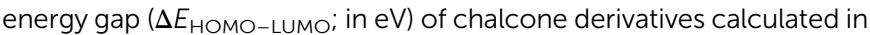
gaseous phase

\begin{tabular}{llll}
\hline Molecule & $E_{\text {Hомо }}(\mathrm{eV})$ & $E_{\text {LUмо }}(\mathrm{eV})$ & $\Delta E_{\text {Hомо-LUмо }}(\mathrm{eV})$ \\
\hline FNPO & -6.786 & -3.429 & 3.357 \\
AFPO & -5.895 & -1.883 & 4.011 \\
FHPO & -0.232 & -0.100 & 0.131
\end{tabular}

carbonyl group and partially in fural group. According to Mulliken charge density of AFPO and FHPO, nitrogen atom $(-0.4495)$ of amine group and oxygen atom $(-0.5929)$ of hydroxyl group have more electron density than oxygen atom $(-0.3135$ and -0.3593$)$ of carbonyl group from AFPO and FHPO respectively. Thus, charge transfer occurs from amine and hydroxyl group to carbonyl group in both AFPO and FHPO. Mulliken charge density parameters have been given in Fig. 7 ESI. $\dagger$

Global chemical reactivity descriptors (GCRD). Global chemical reactivity descriptors are important parameters that give insights into chemical reactivity and stability of the molecules. GCRD parameters such as ionization potential (IP), electron affinity (EA), electronegativity $\chi$, chemical potential $(\mu)$, chemical hardness $(\eta)$, chemical softness $(s)$ and electrophilicity $(\omega)$ for FNPO, AFPO and FHPO have been calculated using the following expressions.

$$
\begin{aligned}
\mathrm{IP} & =-E_{\mathrm{HOMO}}, \mathrm{EA}=-E_{\mathrm{LUMO}}, \chi=(\mathrm{IP}+\mathrm{EA}) / 2, \\
\mu & =-\chi, \eta=(\mathrm{IP}-\mathrm{EA}) / 2, s=1 / 2 \eta
\end{aligned}
$$

The obtained values of GCRD parameters are tabulated in Table 8.

Cyclic voltammetric determination of HOMO-LUMO. Electrochemical properties such as oxidation and reduction potentials of FNPO, AFPO and FHPO were recorded by cyclic voltammetry in acetonitrile medium using $\mathrm{LiClO}_{4}$ as electrolyte 
Table 8 Global reactivity descriptors for FNPO, AFPO and FHPO calculated at the (B3LYP/6-311G(d,p)) level of theory

\begin{tabular}{lrrr}
\hline Molecule & FNPO & AFPO & \multicolumn{1}{c}{ FHPO } \\
\hline Ionization potential, IP (eV) & 6.786 & 1.883 & 0.232 \\
Electron affinity, EA (eV) & 3.429 & 5.895 & 0.100 \\
Electronegativity, $\chi(\mathrm{eV})$ & 5.107 & 3.889 & 0.166 \\
Chemical potential, $\mu(\mathrm{eV})$ & -5.107 & -3.889 & -0.166 \\
Chemical hardness, $\eta(\mathrm{eV})$ & 1.678 & -2.005 & 0.065 \\
Chemical softness, $s(\mathrm{eV})$ & 0.297 & 0.249 & 4.741 \\
Electrophilicity, $\omega(\mathrm{eV})$ & 7.770 & 3.771 & 0.209 \\
& & & \\
\hline
\end{tabular}

Table 9 Electrochemical properties of FNPO, AFPO and FHPO

\begin{tabular}{lccclc}
\hline Compounds & $E_{\mathrm{g}}{ }^{a}$ & $E_{\mathrm{ox}}{ }^{b}$ & $E_{\text {red }}{ }^{c}$ & $E_{\text {HOMO }} / E_{\text {LUMO }}(\mathrm{eV})$ & $E_{\mathrm{g}}$ \\
\hline FNPO & 3.35 & 0.52 & 1.54 & $-4.91 /-5.93$ & 1.02 \\
AFPO & 4.01 & 1.02 & 1.25 & $-5.41 /-5.64$ & 0.23 \\
FHPO & 0.13 & 0.54 & 1.52 & $-4.93 /-5.91$ & 0.98
\end{tabular}

${ }^{a}$ Theoretically calculated values. ${ }^{b}$ Oxidation potential. ${ }^{c}$ Reduction potential in acetonitrile $\left(10^{-3} \mathrm{M}\right)$ containing $0.1 \mathrm{~mol} \mathrm{~L}^{-1} \mathrm{LiClO}_{4}$ with a scan rate of $100 \mathrm{mV} \mathrm{s}^{-1}$.

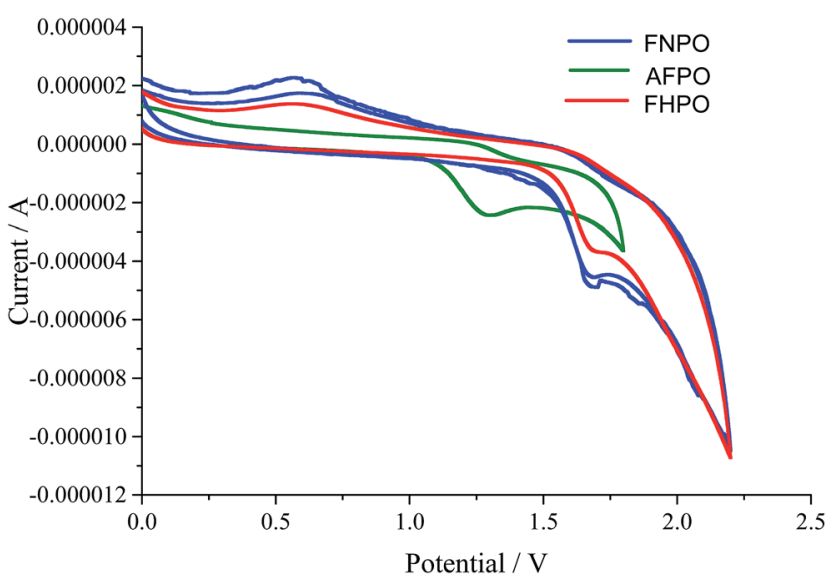

Fig. 7 Cyclic voltammograms of FNPO, AFPO and FHPO $\left(10^{-3} \mathrm{M}\right)$ solvent: acetonitrile; electrolyte: $\mathrm{LiClO}_{4}$; scan rate: $100 \mathrm{mV} \mathrm{s}^{-1}$.

and have been presented in Table 9. HOMO and LUMO energies of the molecules were calculated using oxidation and reduction potential values respectively. ${ }^{65}$ The band gap of FNPO, AFPO and FHPO were calculated from HOMO-LUMO energies. Low band gap of the titled compounds indicate that the molecules were highly reactive. The cyclic voltammograms of FNPO, AFPO and FHPO have been depicted in Fig. 7.

\section{Experimental}

\section{Materials and methods}

All the chemicals and reagents were purchased from Sigma Aldrich Chemical Company, were of analytical grade and used as received. A thin layer chromatography (TLC) using aluminium plates pre-coated with silica gel $60 \mathrm{~F}_{254}$ (mesh) were used to confirm the completion of the reactions. Spots were observed under UV light to ensure the purity of the product. Melting points of synthesized molecules were recorded on electrothermal digital melting point apparatus (Veego, VMPD) and were uncorrected. FTIR data were collected on a Bruker FT/IR spectrometer (Alpha-T) with $\mathrm{KBr}$ pellets. ${ }^{1} \mathrm{H}$ and ${ }^{13} \mathrm{C} \mathrm{NMR}$ spectra were recorded on a Bruker $(400 \mathrm{MHz})$ spectrometer using $\mathrm{DMSO} / \mathrm{CDCl}_{3}$ as solvent and TMS as internal standard. GC-MS/ HRMS data were measured using mass spectrometer (Thermo Trace GC Ultra/Thermo DSQ II). Cyclic voltammetric measurements were carried out on $\mathrm{CH} 1608 \mathrm{E}$ analyser and oxidation and reduction potential is of FNPO, AFPO and FHPO were recorded using a three electrode cell (platinum as the working and counter electrode and scanning calomel electrode as the reference electrode). The scan rate was $100 \mathrm{mV} \mathrm{s}^{-1}$ and the supporting electrolyte was $\mathrm{LiClO}_{4}\left(10^{-3} \mathrm{~mol} \mathrm{~L}^{-1}\right)$ solution in acetonitrile.

Absorption spectra have been recorded on a Shimadzu UVVisible 1800 spectrophotometer. Fluorescence spectra were obtained on Shimadzu RF-5301PC spectrofluorometer. All the solvents of different polarities, used for the study of solvatochromic behavior were of spectroscopic grade. Solutions were prepared in $10^{-5} \mathrm{M}$ concentration to avoid self-absorption and aggregation formation. All plots under solvatochromic and quenching studies were analyzed using Origin 8.0 Professional program.

All theoretical calculations have been performed using Gaussian 09 program. Quantum chemical calculations were employed to determine the ground state dipole moments of FNPO, AFPO and FHPO. ${ }^{47}$

\section{Synthesis}

General procedure for the synthesis of chalcone derivatives. To a solution of furan-2-carbaldehyde $(0.01 \mathrm{~mol})$ and acetophenone derivatives $(0.01 \mathrm{~mol})$ in ethanolic medium $(10 \mathrm{ml})$, $\mathrm{NaOH}(40 \%)$ was added dropwise, and the reaction mixture was stirred at $25{ }^{\circ} \mathrm{C}$ for $24 \mathrm{~h}$. This was kept in a refrigerator and allowed to stand overnight. The crude product was separated by filtration, dried and purified by recrystallization from ethanol.

Characterization of (E)-3-(furan-2-yl)-1-(4-nitrophenyl)prop2-en-1-one (FNPO). Yield 88\%, yellow solid, mp: 151-153 ${ }^{\circ} \mathrm{C}$ (lit. 151-152 $\left.{ }^{\circ} \mathrm{C}\right),{ }^{1} \mathrm{H}$ NMR (400 MHz, DMSO-d $\left.{ }_{6}\right): \delta 6.71(\mathrm{dd}, J=$ $3.3 \mathrm{~Hz}, 1 \mathrm{H}), 7.17$ (d, $J=3.2 \mathrm{~Hz}, 1 \mathrm{H}), 7.51$ (d, $J=15.2 \mathrm{~Hz}, 1 \mathrm{H})$, $7.62(\mathrm{~d}, J=15.6 \mathrm{~Hz}, 1 \mathrm{H}), 7.95$ (d, $J=1.6 \mathrm{~Hz}, 1 \mathrm{H}), 8.27$ (d, $J=$ $4.4 \mathrm{~Hz}, 2 \mathrm{H}), 8.34$ (d, $J=4.4 \mathrm{~Hz}, 2 \mathrm{H}) ;{ }^{13} \mathrm{C} \mathrm{NMR}\left(400 \mathrm{MHz}, \mathrm{CDCl}_{3}\right.$ ): $\delta=113.1,118.1,123.9,129.4,132.2,143.0,145.7,150.1,151.3$, 188.2. IR (KBr), cm ${ }^{-1}: 1659(\mathrm{C}=\mathrm{O}), 1580(\mathrm{C}=\mathrm{C}), 1471\left(\mathrm{NO}_{2}\right.$ asym), 1402 ( $\mathrm{NO}_{2}$ sym), 969 (furan), 812 (Ar-H str). GC-MS m/z: = 243.21 (calcd) 243 (found).

(E)-1-(4-Aminophenyl)-3-(furan-2-yl)prop-2-en-1-one (AFPO). Yield 85\%, yellow solid, mp: $109-110{ }^{\circ} \mathrm{C}$ (lit. $110{ }^{\circ} \mathrm{C}$ ), ${ }^{1} \mathrm{H}$ NMR $\left(400 \mathrm{MHz}, \mathrm{CDCl}_{3}\right): \delta 4.15(\mathrm{~s}, 2 \mathrm{H}), 6.49(\mathrm{dd}, J=3.2 \mathrm{~Hz}, 1 \mathrm{H}), 6.66$ $(\mathrm{d}, J=3.2 \mathrm{~Hz}, 1 \mathrm{H}), 6.69$ (d, $J=8.4 \mathrm{~Hz}, 2 \mathrm{H}), 7.46(\mathrm{~d}, J=15.2 \mathrm{~Hz}$, $1 \mathrm{H}), 7.50(\mathrm{~d}, J=1.2 \mathrm{~Hz}, 1 \mathrm{H}), 7.56(\mathrm{~d}, J=15.2 \mathrm{~Hz}, 1 \mathrm{H}), 7.93(\mathrm{~d}, J=$ $8.8 \mathrm{~Hz}, 2 \mathrm{H}$ ); ${ }^{13} \mathrm{C}$ NMR (400 $\mathrm{MHz}, \mathrm{CDCl}_{3}$ ): $\delta=76.8,77.1,77.4$, $112.6,114.0,115.3,119.5,128.6,129.4,131.1,144.5,151.2$, 152.1, 187.6. IR (KBr), $\mathrm{cm}^{-1}: 3456(\mathrm{~N}-\mathrm{H}), 1648(\mathrm{C}=\mathrm{O})$. HRMS m/ $z:=213.23$ (calcd) $236.0687(\mathrm{M}+\mathrm{Na})$ (found). 
(E)-3-(Furan-2-yl)-1-(2-hydroxyphenyl)prop-2-en-1-one (FHPO). Yield 90\%, yellow solid, mp: 102-104 ${ }^{\circ} \mathrm{C}$ (lit. 103-105 ${ }^{\circ} \mathrm{C}$ ), ${ }^{1} \mathrm{H}$ NMR (400 MHz, $\left.\mathrm{CDCl}_{3}\right): \delta 6.53(\mathrm{dd}, J=3.2 \mathrm{~Hz}, 1 \mathrm{H}), 6.77(\mathrm{~d}, J=$ $3.6 \mathrm{~Hz}, 1 \mathrm{H}), 6.93(\mathrm{~d}, J=16.4 \mathrm{~Hz}, 1 \mathrm{H}), 7.01(\mathrm{~d}, J=9.2 \mathrm{~Hz}, 1 \mathrm{H}), 7.48$ $(\mathrm{d}, J=10 \mathrm{~Hz}, 1 \mathrm{H}), 7.56(\mathrm{t}, J=15.2 \mathrm{~Hz}, 2 \mathrm{H}), 7.68(\mathrm{~d}, J=15.2 \mathrm{~Hz}$, 1H), $7.92(\mathrm{~d}, J=10 \mathrm{~Hz}, 1 \mathrm{H}), 12.89(\mathrm{~s}, 1 \mathrm{H}) ;{ }^{13} \mathrm{C}$ NMR $(400 \mathrm{MHz}$, $\left.\mathrm{CDCl}_{3}\right): \delta=113.0,117.7,118.9,120.1,129.7,131.2,136.4,145.5$, 151.6, 163.6, 193.4. IR (KBr), $\mathrm{cm}^{-1}: 3443$ (O-H), 1641 (C=O). GCMS $m / z:=214.22$ (calcd) 215 (found).

\section{Conclusions}

Photophysical properties of FNPO, AFPO and FHPO were studied in a series of solvents with increasing polarities. Absorption and fluorescence spectra were recorded for eighteen different solvents and the spectral properties of FNPO, AFPO and FHPO were calculated. Large difference of Stokes shift values with increasing polarities for all the molecules have indicated that the bathochromic shift of absorption and fluorescence spectra can be attributed to $\pi-\pi^{*}$ transitions. It suggests that all the molecules were more solvated in singlet excited state than in ground state. The singlet excited state dipole moments were calculated using solvatochromic shift methods and were found to be significantly higher than that of ground state. It confirmed that considerable charge separation occurred between singlet excited state and ground state. This was mainly due to ICT process associated with donor-acceptor moiety. All the molecules were found to be more reactive due to low band gap energy. Therefore, FNPO, AFPO and FHPO can be considered as potential candidates for fluorescent probes, luminescent materials and optoelectronic devices.

\section{Acknowledgements}

We wish to thank Department of IPC, IISc, Bangalore, for providing the facility of Gaussian studies. We thank NMR Centre, IISc, Bangalore, India, for the NMR analysis.

\section{References}

1 (a) A. C. Grimsdale, K. L. Chan, R. E. Martin, P. G. Jokisz and A. B. Holmes, Chem. Rev., 2009, 109, 897; (b) S. M. Kelly, Flat Panel Displays: Advanced Organic Materials, ed. J. A. Connor, The Royal Society of Chemistry, Cambridge, 2000.

2 K. Tainaka, K. Tanaka, S. Ikeda, K. Nishiza, T. Unzai, Y. Fujiwara, I. Saito and A. Okamoto, J. Am. Chem. Soc., 2007, 129, 4776.

3 (a) S. Sreejith, K. P. Divya and A. Ajayaghosh, Chem. Commun., 2008, 2903; (b) T. J. Dale and J. Rebek, J. Am. Chem. Soc., 2006, 128, 4500; (c) S. W. Zhang and T. M. Swager, J. Am. Chem. Soc., 2003, 125, 3420; (d) J. S. Yang and T. M. Swager, J. Am. Chem. Soc., 1998, 120, 11864; (e) J. S. Yang and T. M. Swager, J. Am. Chem. Soc., 1998, 120, 5321; $(f)$ Y. S. Xie, P. Wei, X. Li, T. Hong, K. Zhang and H. Furuta, J. Am. Chem. Soc., 2013, 135, 19119; $(g)$ B. Chen, Y. Ding, X. Li, W. Zhu, J. P. Hill, K. Ariga and Y. Xie, Chem. Commun., 2013, 49, 10136; $(h)$
F. Wang, L. Wang, X. Chen and J. Yoon, Chem. Soc. Rev., 2014, 43, 4312; (i) J. Yin, Y. Kwon, D. Kim, D. Lee, G. Kim, Y. Hu, J.-H. Ryu and J. Yoon, J. Am. Chem. Soc., 2014, 136, 5351.

4 C. W. Mai, Y. Marzieh, N. A. Rahman, Y. B. Kang and M. R. Pichika, Eur. J. Med. Chem., 2014, 77, 378.

5 S. J. Sun, G. Schwarz, R. H. Kricheldorf and T. C. Chang, J. Polym. Sci., Part A: Polym. Chem., 1999, 37, 1125.

6 A. D. Pozzo and A. Dansi, Boll. Chim. Farm., 1979, 118, 239.

7 K. Bowden, A. D. Pozzo and C. K. Duah, J. Chem. Res., 1990, 377.

8 A. O. Doroshenko, A. V. Grigorovich, E. A. Posokhov, V. G. Pivovarenko and A. P. Demchenko, Mol. Eng., 1999, 8, 199.

9 Y. Sato, M. Morimoto, H. Segawa and T. Shimidzu, J. Phys. Chem., 1995, 99, 35.

10 K. Rurack, J. L. Bricks, G. Reck, R. Radeglia and U. R. Genger, J. Phys. Chem. A, 2000, 104, 3087.

11 N. Marcotte, S. F. Forgues, D. Lavabre, S. Marguet and V. G. Pivovarenko, J. Phys. Chem. A, 1999, 103, 3163.

12 B. Mene, K. Kerman, D. Ozkan, P. Kara, A. Erdem and O. Kucukoglu, J. Pharm. Biomed. Anal., 2000, 30, 1339.

13 D. M. Song, K. I. I. Jung, J. I. I. Moon and D. M. Shin, Opt. Mater., 2001, 21, 667.

14 H. K. Hsieh, T. H. Lee, J. P. Wang and C. N. Lin, Pharm. Res., 1998, 15, 39.

15 K. Rama, K. Dharmendra, A. Drishti, D. G. Rinkoo, T. Ragini, K. A. Satish and A. Alka, Eur. J. Med. Chem., 2016, 113, 34.

16 B. Manjunatha, G. K. Nagaraja, K. Reshma, S. K. Peethamber and M. Shafeeullah, RSC Adv., 2016, 6, 59375.

17 M. E. Zwaagstra, H. Timmerman, M. Tamura, Y. T. Wada, K. Onogi and M. Q. Zhang, J. Med. Chem., 1997, 40, 075.

18 O. Mesenzani, A. Massarotti, M. Giustiniano, T. Pirali, V. Bevilacqua, A. Calderlli, P. Canonico, G. Sorba, E. Novellino, A. A. Genazzani and G. C. Tron, Bioorg. Med. Chem. Lett., 2011, 21, 764.

19 C. B. Patil, S. K. Mahajan and S. A. Katti, J. Pharm. Sci. Res., 2009, 3, 11.

20 M. L. Bello, L. D. Chiaradia, L. R. S. Dias, L. K. Pacheco, T. R. Stumpf, A. Mascarello, M. Steindel, R. A. Yunes, H. C. Castro, R. J. Nunes and C. R. Rodrigues, Bioorg. Med. Chem., 2011, 19, 5046.

21 A. N. Romanov, S. K. Gularyan, B. M. Polyak, R. A. Sakovich, G. E. Dobretsov and O. M. Sarkisov, Phys. Chem. Chem. Phys., 2011, 13, 9518.

22 Y. Xue, J. Mou, Y. Liu, X. Gong, Y. Yang and L. An, Cent. Eur. J. Chem., 2010, 8, 928.

23 D. Nagaraja, N. R. Patil, R. A. Kusanur, H. D. Patil and R. M. Melavanki, Int. J. Life Sci. Pharma Res., 2013, 3, L-54.

24 K. Aggarwal and J. M. Khurana, Spectrochim. Acta, Part A, 2015, 143, 288.

25 A. Kawski, B. Kuklinski and P. Bojarski, Chem. Phys. Lett., 2005, 415, 251.

26 J. R. Lombardi, J. Chem. Phys., 1969, 50, 3780.

27 J. Czekella, Z. Electrochem., 1960, 64, 1221.

28 M. P. Hass and J. M. Warman, Chem. Phys., 1982, 73, 35.

29 V. J. Czekella, Chimia, 1961, 15, 26. 
30 S. C. Lee, N. Y. Kang, S. J. Park, S. W. Yun, Y. Chandran and Y. T. Chang, Chem. Commun., 2012, 48, 6681.

31 S. Prabu, R. Nagalakshmi and P. Srinivasan, Spectrochim. Acta, Part A, 2013, 103, 45.

32 Y. Wei, G. Qin, W. Wang, W. Bian, S. Shuang and C. Dong, J. Lumin., 2011, 131, 1672.

33 H. Singh, J. Sindhu and J. M. Khurana, Sens. Actuators, B, 2014, 192, 536.

34 M. Gaber, S. A. El-Daly, T. A. Fayed and Y. S. El-Sayed, Opt. Laser Technol., 2008, 40, 528.

35 H. Singh, J. Sindhu and J. M. Khurana, J. Lumin., 2015, 158, 340.

36 V. Tomeckova, B. Velika, S. Malhotra, M. Revicka and P. Perjesi, Spectrosc. Lett., 2015, 48, 317323.

37 M. Pannipara, et al., Spectrochim. Acta, Part A, 2014, 136, 1893-1902.

38 M. Pannipara, et al., J. Fluoresc., 2014, 24(6), 1629-1638.

39 M. K. Saroj, N. Sharma and R. C. Rastogi, J. Mol. Struct., 2012, 1012, 73-86.

40 C. J. Zheng, S. M. Jiang, Z. H. Chen, B. J. Ye and H. R. Piao, Arch. Pharm., 2011, 344, 689.

41 L. Bilot and A. Kawski, Z. Naturforsch., A: Astrophys., Phys. Phys. Chem., 1962, 17, 621.

42 A. Kawski and J. F. Rabek, Progress in photochemistry and photophysics, CRC Press, 5th edn, 1992.

43 (a) E. Lippert, Zeitscrift fur Electrochemie, 1957, 61, 962; (b) N. Mataga, Y. Kaifu and M. Koizumi, Bull. Chem. Soc. Jpn., 1956, 29, 465.

44 N. G. Bakhshiev, Opt. Spectrosc., 1964, 16, 821.

45 (a) A. Kawski, Acta Phys. Pol., 1966, 29, 507; (b) A. Chamma and P. Viallet, C. R. Seances Acad. Sci., Ser. C, 1970, 27, 1901.

46 C. Reichardt, Chem. Rev., 1994, 94, 2319.

47 R. Kumari, A. Varghese and L. George, J. Lumin., 2016, 179, 518.

48 A. D. Becke, J. Chem. Phys., 1993, 98, 5648.

49 W. H. Melhuish, J. Phys. Chem., 1961, 65229.

50 H. S. Lalithamba, S. R. Manohara, B. Siddlingeshwar and S. Kumaraiah, J. Mol. Liq., 2014, 198, 94.
51 C. Parkanyi and J. J. Aaron, Dipole moments of aromatic heterocycles, in Theoretical Organic Chemistry, ed. C. Parkanyi, Elsevier, 1998, p. 233.

52 J. J. Aaron, M. Maafi, C. Kersebet, C. Parkanyi, M. S. Antonious and N. Motohashi, J. Photochem. Photobiol., A, 1996, 101, 127.

53 A. Adenier, J. J. Aaron, C. Parkanyi, G. Deng and M. Sallah, Heterocycl. Commun., 1996, 2, 403.

54 C. Parkanyi, C. Boniface, A. Sappok-Stang, A. R. Gutierrez, K. Taha-Bouamri and J. J. Aaron, Polycyclic Aromat. Compd., 1999, 13, 55.

55 C. Parkanyi, M. R. Stem-Beren, O. R. Martinez, J. J. Aaron, M. Bulacenau-Macnair and A. F. Arrieta, Spectrochim. Acta, Part A, 2004, 60, 1805.

56 J. J. Aaron, C. Párkányi, A. Adenier, C. Potin, Z. Zajíčková, O. R. Martínez, J. Svoboda, P. Pihera and P. Váchal, J. Fluoresc., 2011, 21, 2133.

57 D. R. Lide, Handbook of Chemistry and Physics, CRC Press, 76th edn, 1995.

58 P. Suppan, Chem. Phys. Lett., 1983, 3272.

59 M. J. Kamlet, J.-L. M. Abboud and R. W. Taft, Prog. Phys. Org. Chem., 1982, 13, 485.

60 J. Catalan, J. Phys. Chem. B, 2009, 113, 5951.

61 K. Guzow, M. Czerwińska, A. Ceszlak, M. Kozarzewska, M. Szabelski, C. Czaplewski, A. Łukaszewicz, A. A. Kubicki and W. Wiczk, Photochem. Photobiol. Sci., 2013, 12, 284.

62 A. Filarowski, M. Kluba, K. C. Boczula, A. Koll, A. Kochel, L. Pandey, W. M. De Borggrave, M. van der Auweraer, J. Catalan and N. Boens, Photochem. Photobiol. Sci., 2011, 9, 996.

63 X. Liu, J. M. Cole and K. S. Low, J. Phys. Chem. C, 2013, 117, 14731.

64 M. E. D. Lestard, D. M. Gil, O. Estevez-Hernandez, M. F. Erben and J. Duquebc, New J. Chem., 2015, 39, 7459.

65 H. Baykara, S. Ilhan, A. Levent, M. S. Seyitoglu, S. Ozdemir, V. Okumus, A. Oztomsuk and M. Cornejo, Spectrochim. Acta, Part A, 2014, 130, 270. 